\title{
Rivalry-Like Neural Activity in Primary Visual Cortex in Anesthetized Monkeys
}

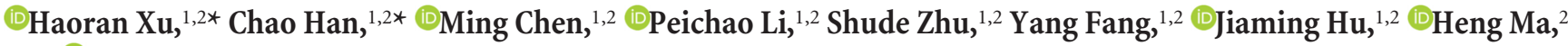 \\ and ${ }^{\circ}$ Haidong $\mathrm{D}$. $\mathrm{Lu}^{2}$ \\ ${ }^{1}$ Institute of Neuroscience, Shanghai Institutes for Biological Sciences, Chinese Academy of Sciences, and University of Chinese Academy of Sciences, \\ Shanghai 200031, China and ${ }^{2}$ State Key Laboratory of Cognitive Neuroscience and Learning, IDG/McGovern Institute for Brain Research, Collaborative \\ Innovation Center for Brain Science, Beijing Normal University, Beijing 100875, China
}

Two incongruent images viewed by the two eyes cause binocular rivalry, during which observers perceive continuous alternations between these two visual images. Previous studies in both humans and monkeys have shown that the primary visual cortex (V1) plays a critical role in the rivalry perception. However, it is unclear whether the rivalry activity observed in V1 relies on conscious influences. Here, we examine the responses of V1 in monkeys under general anesthesia. With intrinsic signal optical imaging and single-trial analysis, alternating activation of ocular dominance columns in V1 was observed during binocularly incongruent stimulation. Left- and right-eye columns exhibited counterphase activation, which were modulated by stimulus features in ways similar to those found in conscious human observers. These observations indicated that binocular rivalry occurs in V1 without consciousness, suggesting that the low-level automatic mechanisms play a more important role than previously believed in handling visual ambiguities.

Key words: anesthetized; attention; binocular rivalry; ocular dominance; optical imaging; striate cortex

\section{Significance Statement}

When visual input is ambiguous, for example, in viewing bistable images, human subjects normally perceive one of the interpretations at a particular moment. Previous studies have shown that both low-level visual processing and high-level attention contribute to the establishment of the final visual perception. However, it is not clear whether attention is indispensable in such a process. Here we show that rivalry-like neural activity persisted in monkey V1 when the monkeys were anesthetized and viewed binocularly incongruent stimuli. Such activity has many key features similar to those observed in conscious human subjects. These findings indicate that low-level visual processes play a critical role in solving visual ambiguity such as binocular rivalry.

\section{Introduction}

Binocular rivalry (BR) occurs when the two eyes view dissimilar images, during which the images perceptually alternate over time. This dissociation between the physical stimulus (stable) and perception (changing) has been widely used as a tool to study visual perception and visual awareness (Blake and Logothetis,

Received Oct. 3, 2015; revised Feb. 3, 2016; accepted Feb. 5, 2016.

Author contributions: H.X., C.H., and H.D.L. designed research; H.X., C.H., M.C., P.L., S.Z., Y.F., J.H., and H.M. performed research; H.X. and C.H. analyzed data; H.X., C.H., and H.D.L. wrote the paper.

This work was supported by the National Natural Science Foundation of China $(31371111,31530029)$ and the Hundred Talent Program of the Chinese Academy of Sciences (H.D.L.) We thank Z. J. He and T. L. Ooi for their contributions to the scientific motivation and methods of analysis and comments on this manuscript. We thank A.W. Roe, R. M. Friedman, and D. Cai for critical comments on this manuscript. Lab members J. Lu, J. W. Pan, J. J. Cai, C. Xu, Z. Yu, C. Fang, Z. L. Ji, X. Y. Cai, and K. Yan provided valuable technical assistance.

${ }^{*} H . X$. and C.H. contributed equally to this work.

The authors declare no competing financial interests.

Correspondence should be addressed to Haidong Lu, School of Brain and Cognitive Sciences, Beijing Normal

University, Beijing 100875, China. E-mail: haidong@bnu.edu.cn.

P. Li's current address: Salk Institute, La Jolla, CA 92037.

DOI:10.1523/JNEUROSCI.3660-15.2016

Copyright $\odot 2016$ the authors $\quad 0270-6474 / 16 / 363231-12 \$ 15.00 / 0$
2002; Alais and Blake, 2005). Previous studies have been fruitful in shedding light on the neural concomitants of BR and its underlying neural mechanisms. Particularly, the primary visual cortex (V1) has been shown to play an important role in the BR process (Polonsky et al., 2000; Tong and Engel, 2001). However, since neural activity in V1 is also modulated by high-level cognitive mechanisms such as voluntary attention (Boynton, 2011; Roeber et al., 2011; Watanabe et al., 2011; Yang et al. 2015), it is unclear whether these cognitive influences are critical for the rivalry in $\mathrm{V} 1$, and whether rivalry-related neural activity exists in the absence of consciousness. To address these questions directly, we examined the response of $\mathrm{V} 1$ in anesthetized monkeys, thereby removing the conscious component of visual processing. If rivalry in V1 requires the high-order conscious process, then we should not be able to see it in anesthetized conditions.

\section{Materials and Methods}

Animal preparation and imaging

A total of five hemispheres from four adult male macaque monkeys (two

Macaca mulatta, two Macaca fascicularis) were examined. Chronic opti- 
cal chambers were implanted from which multiple imaging experiments were performed (Li et al., 2013). All procedures were performed in accordance with the National Institutes of Health Guidelines and were approved by the Institutional Animal Care and Use Committee (Institute of Neuroscience, Chinese Academy of Sciences, and Beijing Normal University).

Each monkey was placed in a stereotaxic apparatus. Monkeys were artificially ventilated and anesthetized with isoflurane (1-2.5\%) during surgery. A 25-mm-diameter circular craniotomy and durotomy were performed (center location, $12.5-16.5 \mathrm{~mm}$ from the midline, $12-17 \mathrm{~mm}$ from posterior bone ridge) to expose visual area V1. The eccentricity of the visual field corresponding to the center of the exposed V1 was 1-5 (visual angle) for different chambers. A 24-mm-diameter imaging chamber was implanted on the craniotomy area, with a transparent artificial dura to protect the exposed cortex.

During the imaging session, anesthesia was switched from isoflurane to thiopental sodium (induction, $10 \mathrm{mg} / \mathrm{kg}$; maintenance, $3 \mathrm{mg} / \mathrm{kg} / \mathrm{hr}$, i.v.; Ts'o et al., 1990; Li et al., 2013). Anesthetic depth was assessed continuously via monitoring heart rate (140-200 beats/min), end-tidal $\mathrm{CO}_{2}$ $(4-5 \%)$, blood oximetry $(\sim 100 \%)$, and in some experiments EEG (stage III anesthesia; Pichlmayr et al. 1987). Rectal temperature was maintained at $38^{\circ} \mathrm{C}$. These signals reflected a stable anesthesia depth at the dosage described above. Only occasionally did we observe signs of light anesthesia (once in all the experiments reported here), and a top-up of $10 \mathrm{mg}$ ( 1 $\mathrm{ml}$ ) of thiopental sodium was injected intravenously. Animals were paralyzed (vecuronium bromide; induction, $0.25 \mathrm{mg} / \mathrm{kg}$; maintenance, $0.05-0.1 \mathrm{mg} / \mathrm{kg} / \mathrm{hr}$, i.v.) and respirated. Pupils were dilated (atropine sulfate $1 \%$ ), and eyes fit with contact lenses of appropriate curvature to focus on a stimulus screen $57 \mathrm{~cm}$ from the eyes. The brain was stabilized with agar and imaged through a cover glass. Images of cortical reflectance changes (intrinsic hemodynamic signals) corresponding to local cortical activity were acquired (Imager 3001, Optical Imaging) with $632 \mathrm{~nm}$ illumination. Image size was either $504 \times 504$ or $540 \times 654$ pixels representing either a $19 \times 19$ or $20 \times 24 \mathrm{~mm}$ field of view.

\section{Dichoptic view and binocular alignment}

For dichoptic stimulation, a pair of $7^{\circ}$ prisms were used to diverge the two eyes' views horizontally so two patches of stimuli for two eyes could be presented on the left and right halves of the CRT screen (width, $40^{\circ}$; height, $30^{\circ}$ ). The visual field locations of the exposed $\mathrm{V} 1$ were determined with an imaging procedure (Lu et al., 2009). Briefly, single horizontal and vertical bars at different screen locations were presented to each eye alone (through the prism), and the V1 retinotopic activation was measured. With this method we could achieve a precision of $0.1^{\circ}$, which means that a stimulus shift of $0.1^{\circ}$ was detectable from V1 activation (Lu et al., 2009). Although the paralytic drug greatly reduced spontaneous eye movement, the eyes occasionally had some slow drifts. Thus, during the rivalry imaging, eye positions were checked every one hour after initial measurement using the procedures described above, and stimulus locations were adjusted when an eye drift was detected.

\section{Visual stimulus}

Visual stimuli were created using ViSaGe (Cambridge Research Systems) and displayed on a calibrated 21 inch CRT monitor (SONY CPD-G520) running at a $100 \mathrm{~Hz}$ refreshing rate.

Stimuli for rivalry imaging were two square patches presented on the left and right halves of the CRT monitor. Depending on the location of exposed V1, the side length of the patches was selected from $1.5-3.5^{\circ}$ (mean size, $2.6^{\circ}$ ) to activate the exposed V1. These two patches of stimuli were converged by the prisms to form a fused view (Fig. 1A). The rest of the screen was black (luminance, $0.87 \mathrm{~cd} / \mathrm{m} 2$ ). Due to slight differences in imaging chamber locations, stimulus patch size was adjusted for each case so that the activated V1 region was large enough to cover most of the exposed V1 region.

A typical imaging run consisted of six stimulus conditions: two binocularly incongruent (BI) conditions, two stimulus-alternation conditions, one plaid/RD condition, and one blank condition. Within each run, the order of these stimuli was randomized. Each condition lasted 60 or $55 \mathrm{~s}$. Imaging duration also included a $0.5 \mathrm{~s}$ baseline imaging before stimulus presentation. A total of $60.5 \mathrm{~s}$ (or $55.5 \mathrm{~s}$ ) of images were collected at a $4 \mathrm{~Hz}$ frame rate. Between two stimulus conditions, there was a 25 - to 40 -s-long interstimulus interval (ISI) period, during which two stationary random dot $(\mathrm{RD})$ patches (see below, ISI stimulus) were displayed at the corresponding stimulus regions to prevent abrupt luminance changes. These $\mathrm{RD}$ patches stayed on during the $0.5 \mathrm{~s}$ of baseline imaging.

In the BI conditions, two patches of square-wave gratings (half images) were presented to the two eyes simultaneously. One patch was a $45^{\circ}$ oriented red grating; the other patch was a $135^{\circ}$ green grating. Since signal amplitude is crucial in the following single-trial analysis, grating parameters were optimized to drive V1 response (spatial frequency (SF), 2.5 cycles/degree; drifting temporal frequency (TF), 7.5 cycles/s, equivalent to a speed of $3 \%$; contrast, $100 \%$; duty cycle, 0.2 , i.e., $20 \%$ red or green and $80 \%$ black; drifting direction, orthogonal to the grating orientation and randomly chosen from two possible directions for each trial). The luminance for black bands was $0.87 \mathrm{~cd} / \mathrm{m}^{2}$, and the luminance for red and green bands was adjusted for each animal to achieve balanced eye stimulation (described below in Color balance test). Red and green CIE (Commission Internationale de l'Eclairage) values were $(0.609,0.328)$ and $(0.277,0.590)$. BI stimuli were displayed continuously during the BI imaging trial. Two BI conditions were tested in each run, in which the left and right half images were switched between conditions.

In the stimulus-alternation conditions, the two eyes were stimulated alternatively. The two monocular images were the same as the two half images used in the $\mathrm{BI}$ conditions (red $45^{\circ}$ gratings and green $135^{\circ}$ gratings). An image was presented to one eye for $2 \mathrm{~s}$, and then the other image was presented to the other eye for $2 \mathrm{~s}$. The $4 \mathrm{~s}$ cycle was repeated 15 times in a $60 \mathrm{~s}$ imaging trial. Similar to the two BI conditions, two stimulusalternation conditions were tested, in which left- and right-eye images were switched.

In the plaid condition, two identical plaid half images were presented to the two eyes simultaneously for $60 \mathrm{~s}$. The plaid half images were created by overlaying the two half images used in the BI conditions. The color for overlapping regions of two gratings was a simple combination of the red and green colors, which is yellow.

In the $\mathrm{RD}$ condition, two $\mathrm{RD}$ half images were presented to the two eyes simultaneously for $60 \mathrm{~s}$. Each RD pattern was made of dots sized $0.15 \times 0.15^{\circ}$ with either red or green colors (same colors as in the BI condition). Dot density was $20 \%$ (percentage of stimulus area covered with dots). The two RD patches were generated independently (in 10 trials) or used the same pattern (in 39 trials) and refreshed simultaneously at $12.5 \mathrm{~Hz}$ frequency during the $60 \mathrm{~s}$ presentation.

In the blank condition, the ISI stimulus was removed after $0.5 \mathrm{~s}$ of baseline imaging, and the whole screen was black (luminance, $0.87 \mathrm{~cd} /$ $\mathrm{m}^{2}$ ) during the $60 \mathrm{~s}$ imaging period.

ISI stimulus. During the ISI, two identical patches of RD patterns, the same as those used in the RD condition, were displayed. The ISI RD patches were stationary without refreshing.

Full-screen stimulus. Basic functional maps [e.g., ocular dominance (OD) and orientation maps] were used to determine the eye-specific and orientation-specific domains in V1 (described in Functional domain identification session, below). Stimuli used for obtaining these maps were full-screen $\left(40 \times 30^{\circ}\right)$ square-wave gratings (SF, 1.5 cycle/degree; speed, $8 \%$; duty cycle, 0.2 ; contrast, $100 \%$; mean luminance, $16.1 \mathrm{~cd} / \mathrm{m}^{2}$ ) presented at four different orientations (Li et al., 2013). Each stimulus was presented for $4 \mathrm{~s}$, during which cortical responses were imaged. A pair of mechanical shutters was placed in front of the two eyes to achieve monocular stimulation (Li et al., 2013).

Color balance test. In BI condition, imbalance of red/green luminance in two half images caused biased eye dominance. Different animals also had different balanced red/green luminance. Thus, for each chamber, we first tested a series of the red/green luminance ratios and found the one caused a balanced V1 activation. This value was then used in the subsequent BI stimulation. The stimuli in the color balance test were red/green half images similar to those in the BI conditions, with different red/green luminance combinations (Fig. 2C). The stimulus was presented for $4 \mathrm{~s}$, during which $\mathrm{V} 1$ responses were imaged. When the red and green gratings were equally effective (balanced), such stimulus caused equal probability of dominance for the two eyes. When the red and green gratings were not equally effective, it 
A

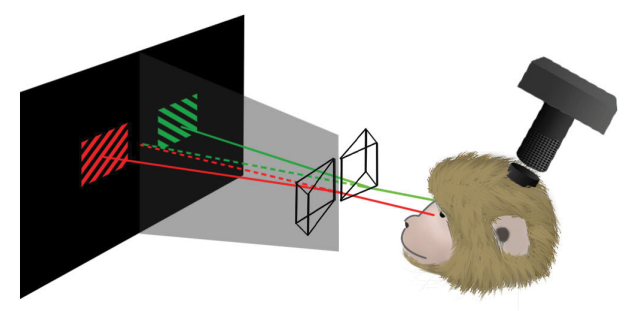

C

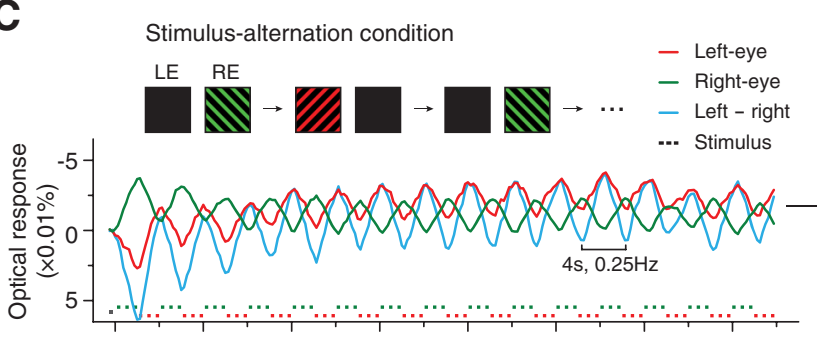

F

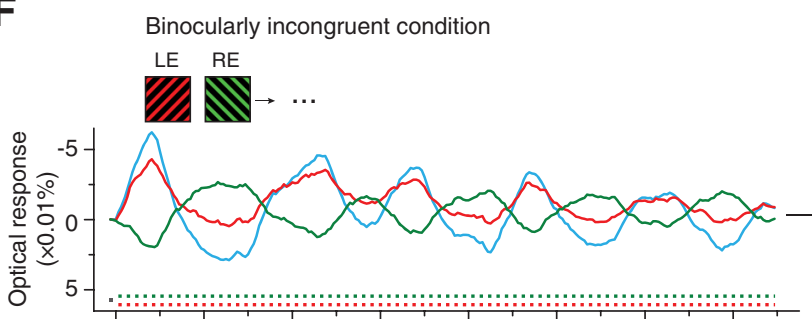

I

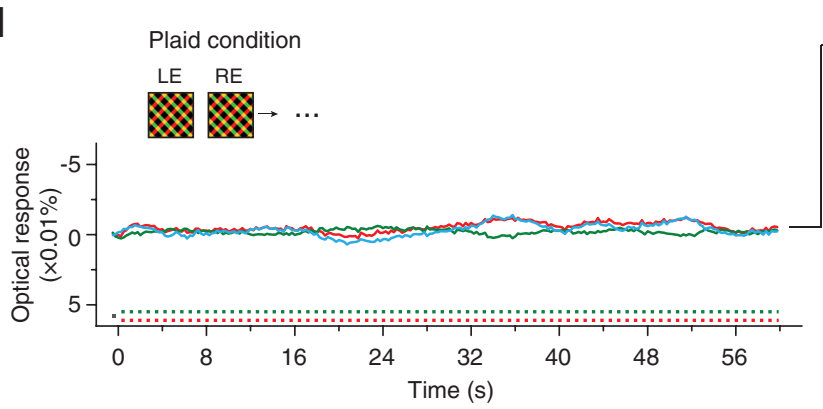

B

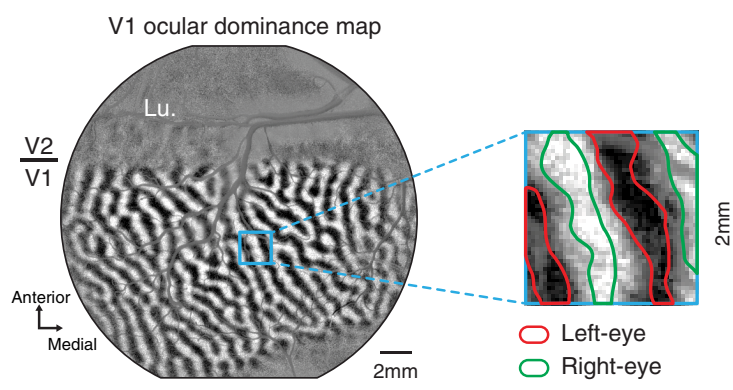

D

E

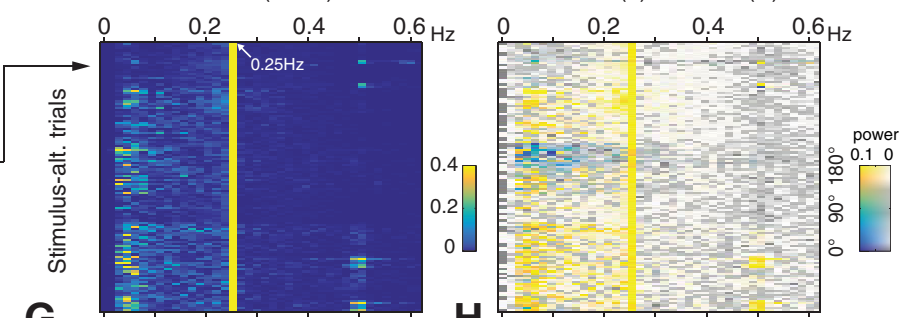

G

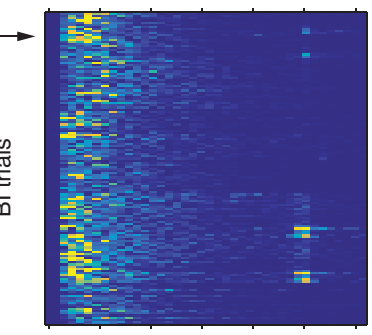

$\mathrm{H}$
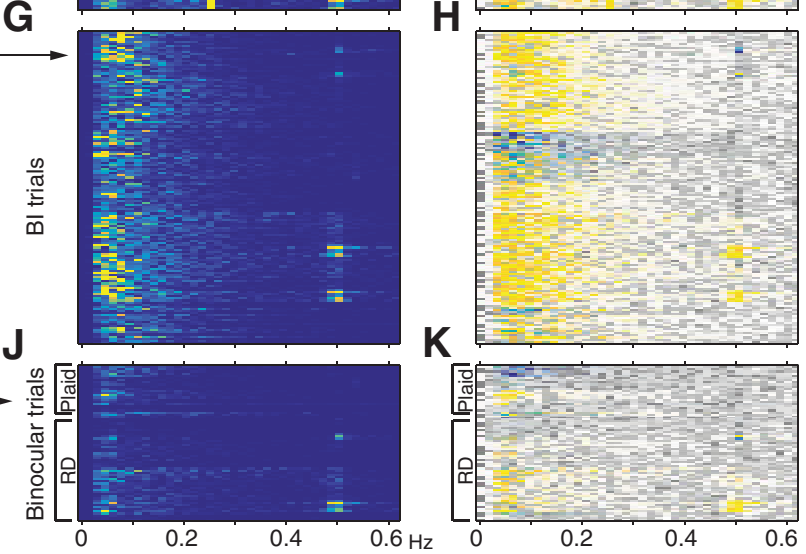

K
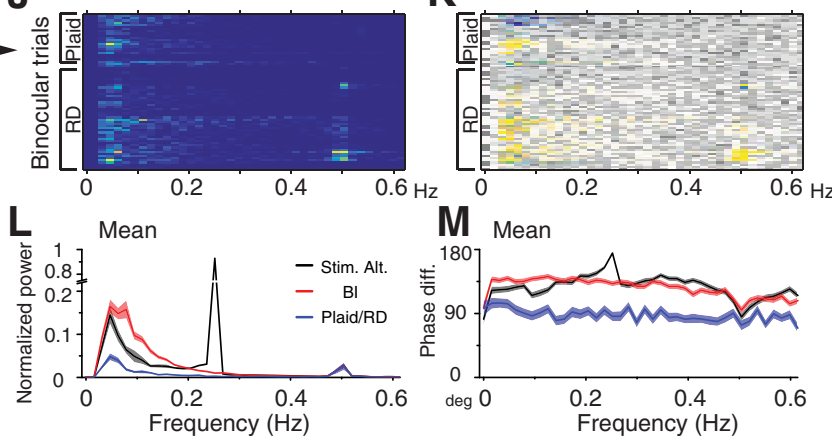

Figure 1. Optical imaging of the V1 response to a binocularly incongruent stimulus. $A$, Illustration of the visual stimulus and imaging setup. $B$, An OD map in V1 obtained by large monocular stimulations. A2 $\times 2 \mathrm{~mm}$ region (blue frame) is magnified to show the outlines of left-eye and right-eye columns (inset). The actual stimulus ROl activated by BI stimulus normally occupied most of V1 surface in the chamber. Lu, Lunate sulcus. C, Example single-trial time courses of V1 left-eye (LE) pixels (red), right-eye (RE) pixels (green), and the difference between these two (cyan) in one stimulus-alternation trial. Negative was plotted upward because the negative optical-signal indicates a response increase. The response alternations follow the 4-s stimulus alternations (bottom traces). $\boldsymbol{D}$, FFT power spectrums of $L-R$ time courses (e.g., cyan curve in $\boldsymbol{C}$ ) for 128 stimulus-alternation trials. Each row represents one trial. $\boldsymbol{E}$, Phase differences between left-eye responses and right-eye responses for the same 128 trials shown in $\boldsymbol{D}$, with saturation of the color code weighted by the corresponding power amplitudes. $\boldsymbol{F}-\boldsymbol{H}$, Similar to $\boldsymbol{C}-\boldsymbol{E}$, for BI data. Example time courses from a single-trial BI response show continuous fluctuations $(\boldsymbol{F})$. Frequency analysis of all $B \mathrm{BI}$ trials $(n=148)$ shows a distribution of fluctuation energy at $0.03-0.2 \mathrm{~Hz}(\boldsymbol{G})$. Responses in the left- and right-eye domains are mostly antiphase $(\boldsymbol{H}) . \boldsymbol{I}-\boldsymbol{K}$, Similar to above, example trial and population results for two binocular control conditions (plaid and RD condition). Time courses of the responses to binocular plaid patterns are mostly flat $(\boldsymbol{I})$. Population frequency analysis $(n=74)$ shows an absent of alternations $(\boldsymbol{J})$ and only small interocular phase differences $(\boldsymbol{K})$. $\boldsymbol{L}, \boldsymbol{M}, \mathrm{Trial}$-averaged mean $\left( \pm\right.$ SEM) power spectrums $(\boldsymbol{L})$ and phase spectrums $\left(\boldsymbol{M}\right.$, nonweighted) show differences between Bl and plaid/RD conditions (two-way ANOVA, $\boldsymbol{L}, F_{(1,8800)}=217.04, p<0.001 ; \boldsymbol{M}, F_{(1,8800)}=$ $915.79, p=0)$.

caused stronger dominance for one eye. In such an imbalance condition, switching the two half images caused the other eye to become more dominant. For each red/green pair, we calculated a subtraction map for these two switched conditions during the imaging experiments. An OD pattern was observed in the online subtraction map when two colors were not balanced (normally after averaging of five or six trials). We then varied the luminance value of the red gratings (either increase or decrease, depending on the sign of the OD map) while keeping the green gratings the same. This procedure was repeated until no/minimum OD patterns were observed in the online subtraction maps. The final red/green values were used in the following BI and plaid conditions. The luminance range of red color tested was 5.6-16.9 $\mathrm{cd} / \mathrm{m}^{2}$; the green color luminance, fixed in one experiment but variable for different experiments, ranged from 27.5 to $55 \mathrm{~cd} / \mathrm{m}^{2}$. The balanced point of the red/green equal luminance ratios ranged between 0.15 and 0.23 for different monkeys.

Monocular boundary contour stimulus. Psychophysics studies have shown that many stimulus features including spatial context modulate BR eye dominance (Blake and Logothetis 2002). One important and dramatic spatial context effect is the monocular boundary contour $(\mathrm{MBC})$ effect, in which the half image containing a boundary 


\section{A}
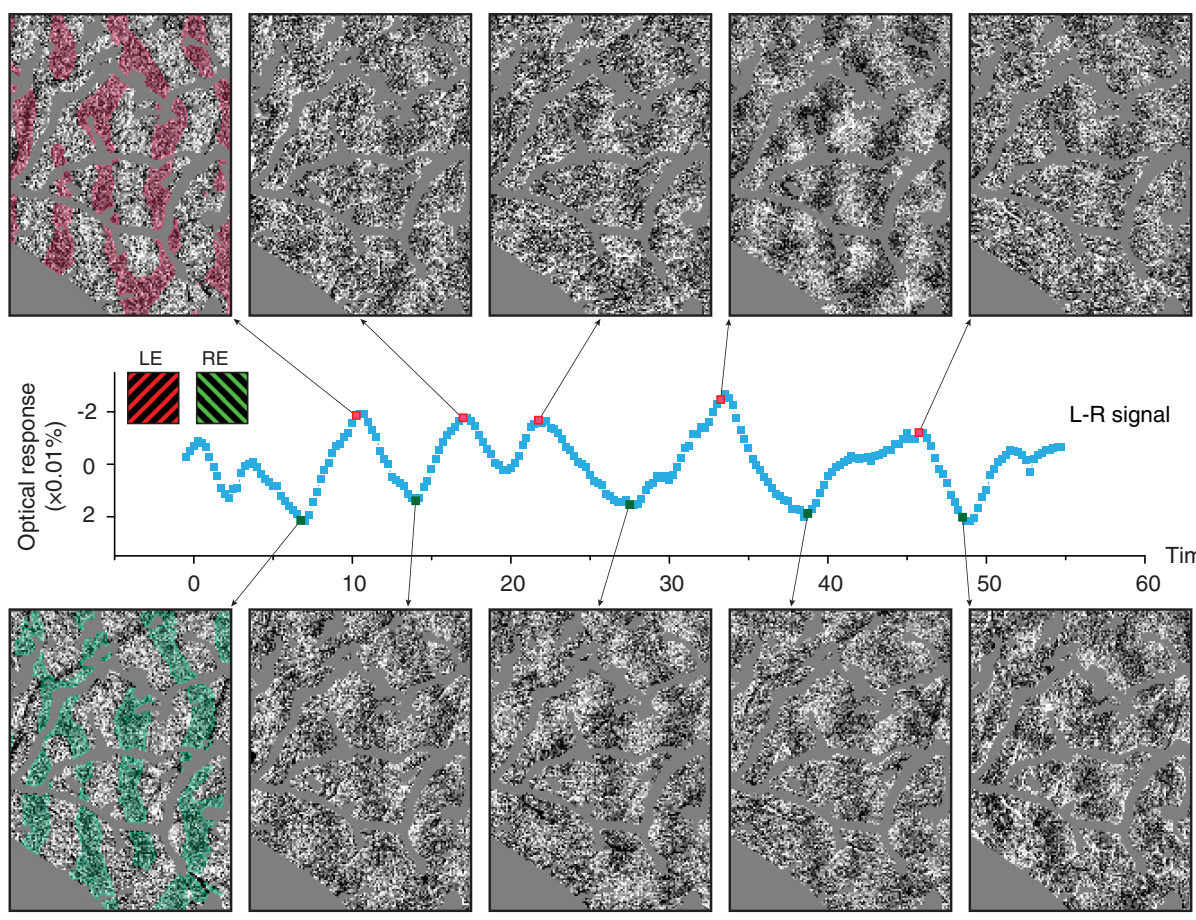

B

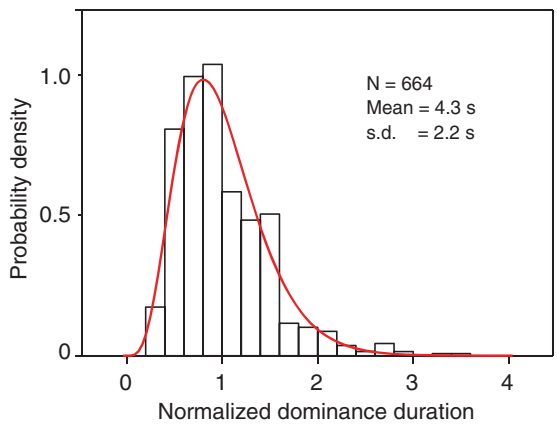

D

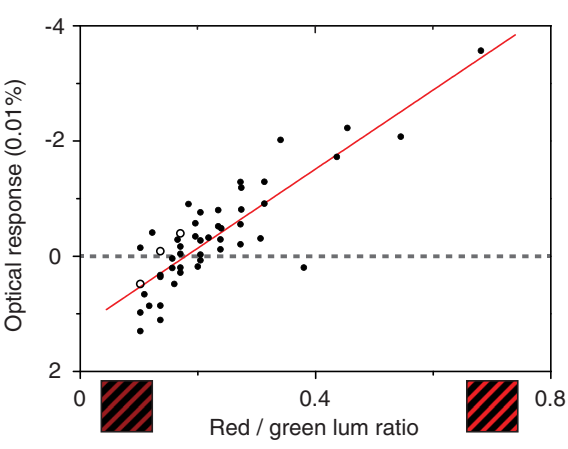

C

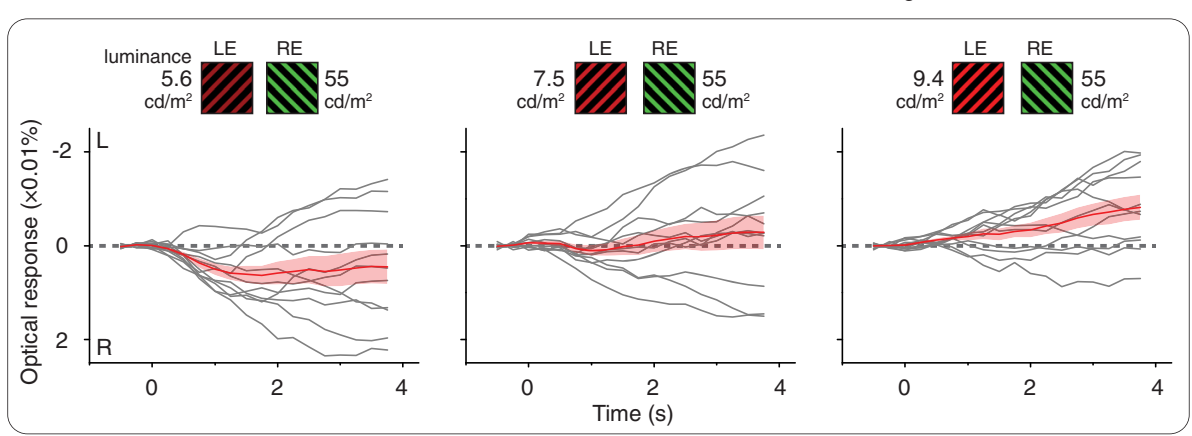

Figure 2. OD fluctuation and modulation. $A$, Single-trial frames imaged during BI stimulation exhibited complementary OD patterns in locally averaged frames (top and bottom). Red and green transparent shades are known left-eye (LE) and right-eye (RE) columns, respectively, superimposed for comparison. Each example image is an average of neighboring 3 imaging frames $(0.75 \mathrm{~s})$ after subtracting a baseline averaged from 49 neighboring frames (12s). Blood vessel regions were replaced with gray color. Also see Movies 1 and 2.B, Similar to psychophysical findings (Levelt, 1967), the distribution of V1 eye dominance durations can be fit by a gamma function (red curve, $R^{2}=0.94$ ). $C, L-R$ time courses for three different binocularly incongruent conditions (right-eye biased, balanced, left-eye biased). Each condition contained 114 s trials (gray curves) and their mean ( \pm SEM; red curves). $D$, Mean L-R values as a function of the relative stimulus strength from all cases. Each data point represents a test similar to a panel in $C$. Open circles are examples shown in $C$. Red line, Data fit with a linear function $\left(R^{2}=0.73\right)$.

contour gets enhanced predominance (Ooi and He, 2006). We also included MBC stimuli in our imaging study to see if V1 eye dominance activity is modulated in a way similar to the perceptual observation.

In $\mathrm{MBC}$ conditions, square-wave luminance gratings were used (SF, 3 cycles/degree; drifting TF, 2 cycles/s; contrast, 100\%; duty cycle, 0.3 ; drifting direction, orthogonal to the grating orientation and randomly chosen from two possible directions for each trial). Both half images had grating backgrounds of the same orientation and size $\left(5 \times 5^{\circ}\right.$ or $\left.7 \times 7^{\circ}\right)$. One half image comprised a grating disk $\left(1.5\right.$ or $2^{\circ}$ diameter) whose orientation was orthogonal to its grating background (Fig. 3A). The two half images thus had rival centers and the same backgrounds. For two types of grating orientations $\left(45\right.$ or $\left.135^{\circ}\right)$ and two types of eye specificity (MBC disk in left or right eye), a total of four conditions were tested (Fig. $3 A$ ). Imaging duration was $4.5 \mathrm{~s}$ for each trial. 
A
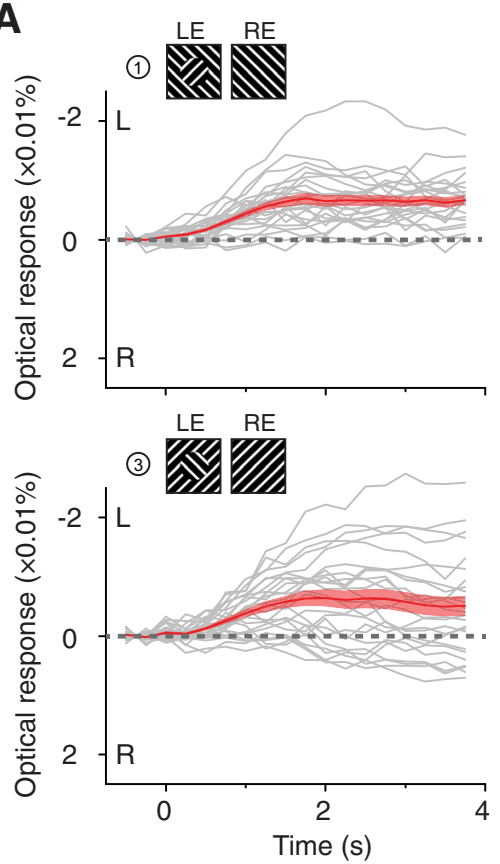

B

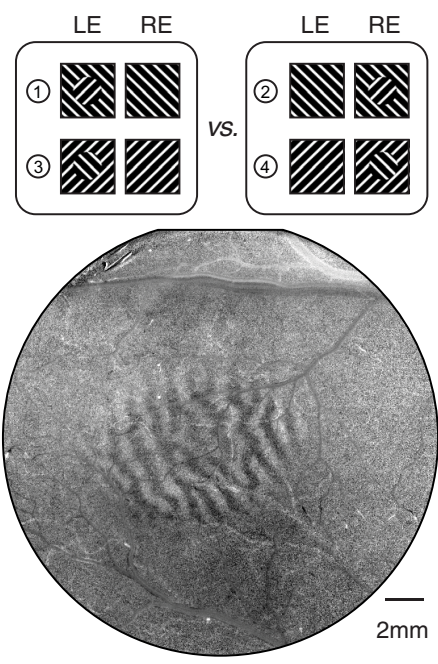

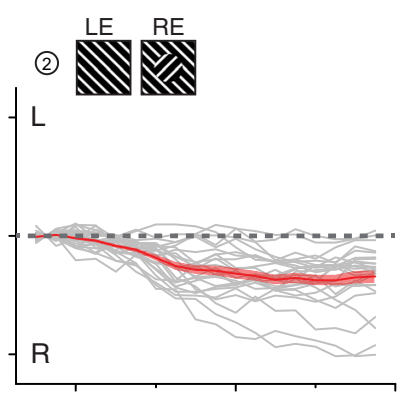

(4)

$-\mathrm{L}$

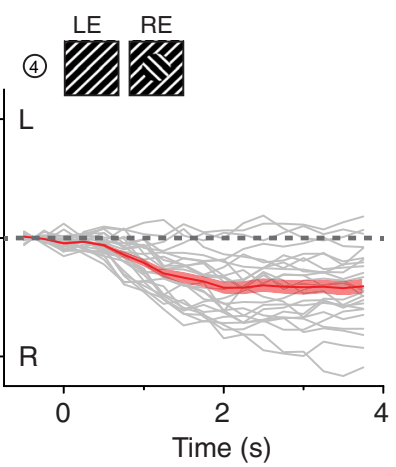

C
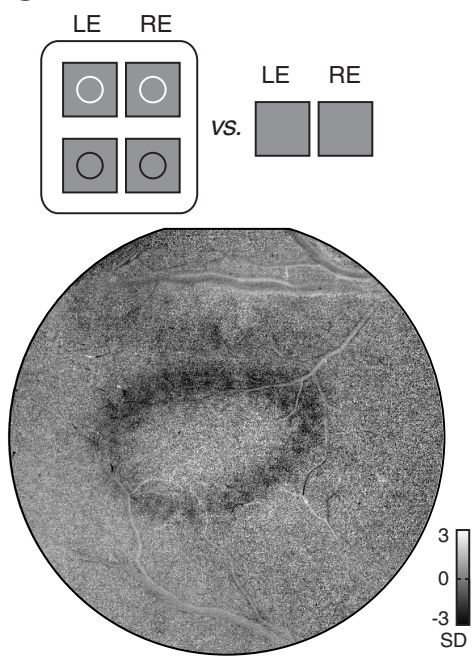

Figure 3. V1 alternation modulated by boundary contours. $A$, Response time courses for four different configurations of $M B C$ stimuli having rivaling centers. The gray curves are individual L-R time courses $(n=25)$ calculated from the V1 region corresponding to the central disk. The red curves are the average time courses ( \pm SEM). V1 activation clearly showed biases toward the eye viewing a boundary contour. $\boldsymbol{B}, A n O D$ pattern was revealed in the contrast map comparing left-eye (LE) and right-eye (RE) MBC conditions (the same data as in $\boldsymbol{A}$ ). $\boldsymbol{C}$, V1 retinotopic representation of a circular contour (line width $0.1^{\circ}$ ) confirms that the eye dominance in $\boldsymbol{B}$ was within the disk representation.

\section{Imaging data analysis}

$d R / R$ values. Raw pixel values were first transformed to $d R / R$ values (percentage change values) using the following function: $d R / R=$ $\left(R-R_{0}\right) / R_{0}$, in which $R$ is the pixel value in each frame and $R_{0}$ is the prestimulus pixel value imaged right before the stimulus onset (averaged from the two initial imaging frames). Subsequent analyses were based on $d R / R$ values unless specified otherwise. The BI image frames were spatially filtered with low- and high-pass filters (disk kernels, 2 and 50 pixels, respectively).

$T$ test maps ( $t$ maps) were obtained by a pixel-by-pixel paired $t$ test between response frames in the two stimulus conditions (Li et al., 2013). $T$ maps take into account trial-to-trial variations in the identification in signal differences, and thus are more reliable than the regular subtraction maps. Details on the $t$ test calculation and comparison with subtraction maps have been described previously (Li et al., 2013). Basic functional

maps included OD maps obtained by comparing left-eye response frames and right-eye stimulation frames. Orientation maps were determined by comparing frames obtained from two orthogonal orientation conditions. Basic functional maps (Figs. 1B, 5A) were filtered with a low-pass filter (disk kernel, 2 pixels) and a high-pass filter (Gaussian kernel, 50 pixels) and clipped at $3 \mathrm{SD}$ for display. Maps shown in Figures 3, $B$ and $C$, and 4 were not filtered. OD and orientation maps were used for identifying domain-specific pixels (see Functional domain identification session, below).

Stimulus region of interest in V1. For each experiment, a stimulus region of interest (ROI) in V1 was identified based on an OD map obtained with monocular stimulus patches that were the same size as those in the BI conditions. Pixels overlying large blood vessels were not included for quantitative analysis. This stimulus ROI contains, on average, 90,000 pixels. Subsequent pixelaveraging quantification included only pixels within this region.

Functional domain identification. An OD map contains white, black, and gray pixels. Based on the pixel value distribution, we obtained two threshold values that equally divided all ROI pixels into three groups [left eye $(\mathrm{L})$, right eye $(\mathrm{R})$, and $\mathrm{OD}$ transition zone $\left(\mathrm{T}_{\mathrm{OD}}\right)$; Fig. $\left.1 B\right]$. Each type of domain contains one-third of the pixels in the stimulus ROI. Similarly, based on a 45 versus $135^{\circ}$ orientation map, we also equally divided the stimulus ROI into three types of domains $\left[45^{\circ}, 135^{\circ}\right.$, and $\mathrm{T}_{\text {ori }}$ (orientation transition zone)]. Together we obtained nine compartments by combining these two types of divisions, including $\mathrm{L} 45, \mathrm{LT}_{\text {ori }}, \mathrm{L} 135, \mathrm{~T}_{\mathrm{OD}} 45$, $\mathrm{T}_{\mathrm{OD}} \mathrm{T}_{\text {ori }}, \mathrm{T}_{\mathrm{OD}} 135, \mathrm{R} 45, \mathrm{RT}_{\text {ori }}$, and R135 (Fig. $5)$. For simplicity, only four of the nine compartments are shown in Figure 5, B and $C$.

Pixel-averaged time course. A domainspecific, pixel-averaged time course was obtained from single-trial $d R / R$ frames by averaging all pixel values within one type of functional domains frame by frame. For example, the time-dependent change of averaged L45 domain value was referred to as L45 time course. Two types of pixel-averaged time courses were shown: single-domaintype time courses (e.g., time courses in Figs. $5,6)$ and subtractions between two domain types (e.g., time courses in Figs. 1-3). Left- or right-eye time courses in Figure 1 are subtraction time courses between left- or right-eye domains and $\mathrm{T}_{\mathrm{OD}}$ domains, respectively.

Frequency spectrum analysis. To assess fluctuation frequency information in stimulus-alternation, BI, plaid, and RD trials, single L-R signal time courses were analyzed with a fast Fourier transform (FFT; Fig. 1D). Each raw L-R signal was first detrended by subtracting a fourth polynomial fitting (Tanabe et al., 2002; Friman et al., 2004). Each FFT power spectrum was then normalized to its frequency response at $0.25 \mathrm{~Hz}$ in the stimulus-alternation condition. To analyze the relative response phases between left- and right-eye domains, the FFT analysis was performed on the two monocular signal time courses $\left(\mathrm{LT}_{\mathrm{OD}}\right.$ and $\left.\mathrm{RT}_{\mathrm{OD}}\right)$. A phase difference spectrum was then calculated by subtracting of these two phase spectrums (Fig. 1E). In the phase spectrum plot, the phase differences were color coded in the HSV (hue, saturation, value) color table, and the 
A

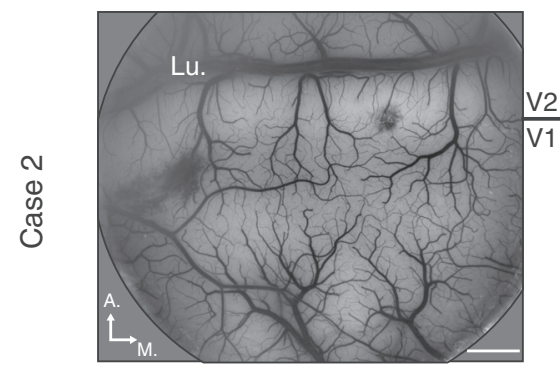

D

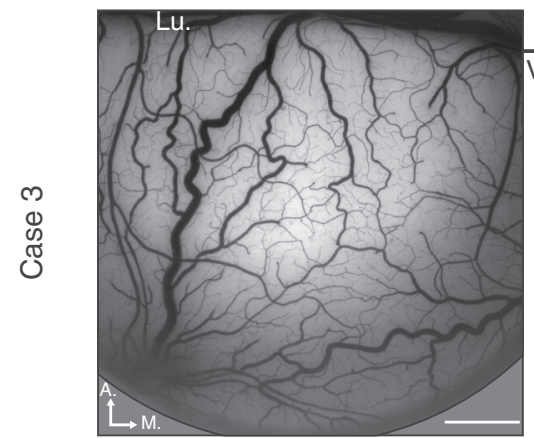

G

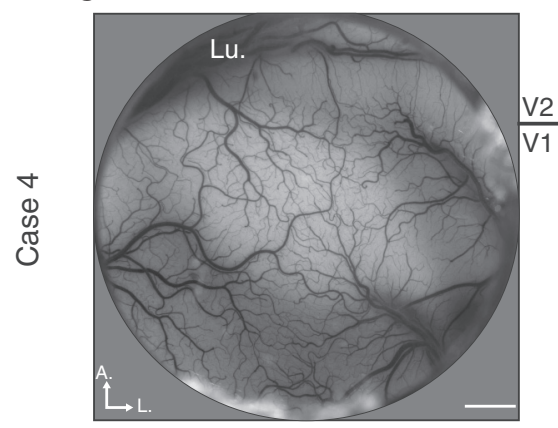

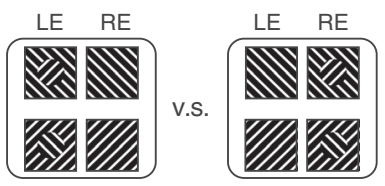

B

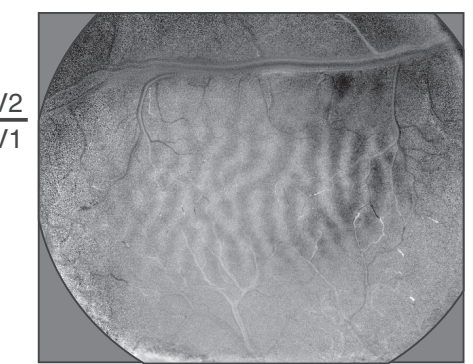

E

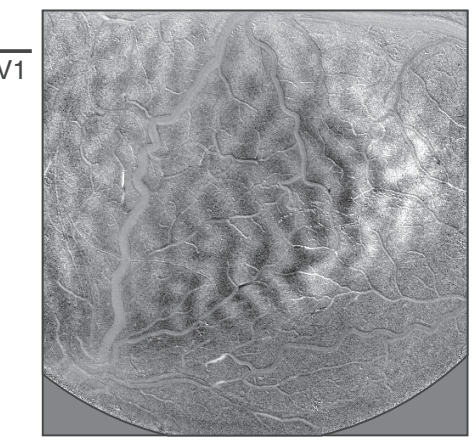

H

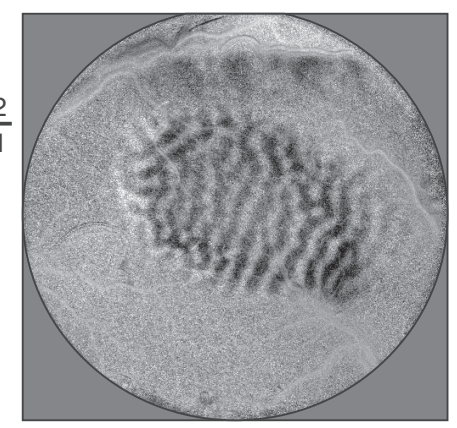

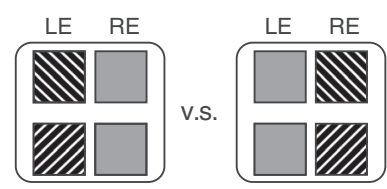

C

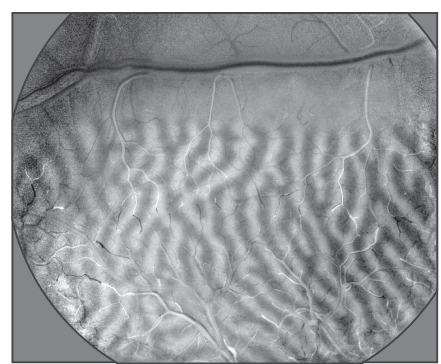

F

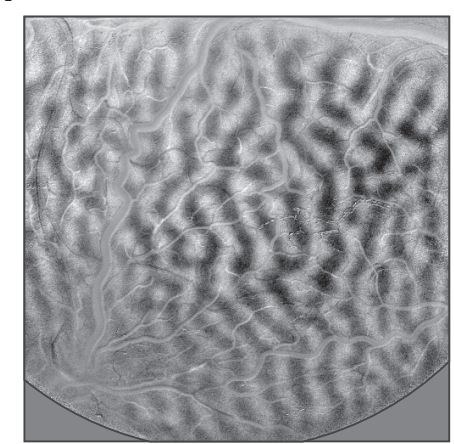

I

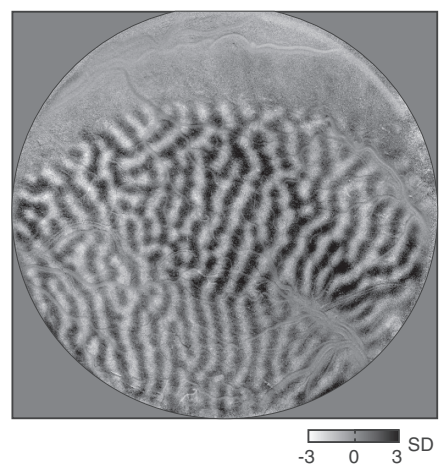

Figure 4. MBC imaging cases. Three more cases imaged with MBC stimuli are shown. The three columns are surface blood vessel maps (left), $M B C$ maps (middle) obtained by comparing MBC in left-eye (LE) conditions and $\mathrm{MBC}$ in right-eye ( $\mathrm{RE}$ ) conditions (similar to Fig. $3 B$ ), and OD maps (right) obtained by comparing left-eye and right-eye monocular stimulation conditions (similar to Fig. 1B). $\boldsymbol{A}-\boldsymbol{C}$, Images from Case 2, in which MBC stimuli was a $1.5^{\circ}$ disk on a $5^{\circ}$ background. $\boldsymbol{D}-\boldsymbol{F}$, Images from Case 3 , in which $\mathrm{MBC}$ stimuli was also a $1.5^{\circ}$ disk on a $5^{\circ}$ background. In this case, the exposed V1 cortex was closer to the fovea region, and thus the same $1.5^{\circ} \mathrm{MBC}$ disk corresponded to a larger V1 map. G-I, Images from Case 4 , in which MBC stimuli was a $1.5^{\circ}$ disk on a $7^{\circ}$ background. All three cases show a biased eye activation elicited by the MBC disk. No filtering was applied to these maps. Lu, Lunate sulcus. Scale bars: $2 \mathrm{~mm}$.

saturation values were weighted by the power amplitudes of corresponding frequencies.

V1 eye dominance duration. V1 eye dominance durations (Fig. 2B) were measured from the L-R signal time courses. We first located the transition time points, where eye dominance switches between the two eyes, by determining the peaks and troughs of the $\mathrm{L}-\mathrm{R}$ time courses' first derivatives. Time courses and their derivatives were smoothed before the peak detection (Gaussian convolution; kernel size, 1.75-2.25 s; SD, 0.3 s). A dominance phase was then defined as the period between two adjacent transition points. For dominance phases found in the above procedures $(n=2695)$, many low-amplitude phases were likely caused by noise. In the plaid and RD data analyzed with the same procedures, we found $95 \%$ of the peaks/troughs to have an amplitude smaller than $0.01 \%$ (reflectance change). Thus, we used $0.01 \%$ as a threshold and only analyzed peaks and troughs having amplitudes larger than $0.01 \%$ ( 664 dominance phases, $25 \%$ of the 2695 phases originally detected). The mean and SD of these dominance durations were calculated. A V1 dominance duration distribution was calculated after normalization within each case (divided by the case mean). A gamma probability density function was fit to the normalized V1 dominance duration data using maximum likelihood estimates (Fig. $2 B): y=f(x \mid a, b)=\left[1 /\left(b^{a} \Gamma(a)\right)\right] x^{a-1} e^{-x / b}$, where $x$ is the phase duration, $\Gamma$ is the gamma function, and $a$ and $b$ are the fitting parameters.

Peak-aligned average response. To evaluate the relative response strengths in different functional domains to stimulus-alternation and BI stimuli, we calculated average domain responses as follows (using L45R135 stimulus-alternation data as example). For all L45R135 stimulus-alternation trials, L45 time courses were first calculated, and their peaks were identified (threshold, $0.005 \%$ reflectance change, half of the threshold used for L-R time courses in the dominance duration calculation). We used these peak times as reference points and extracted 


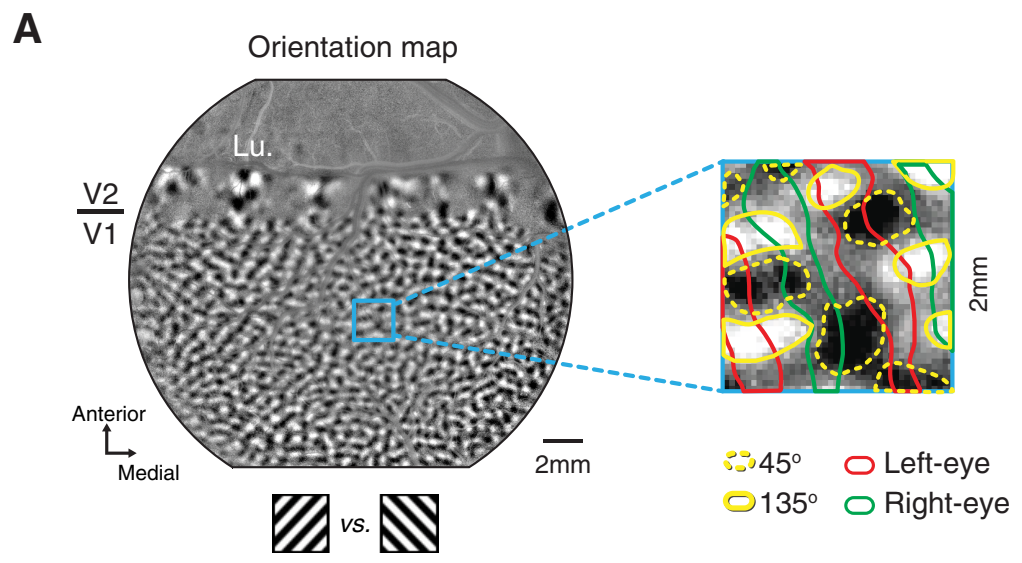

B

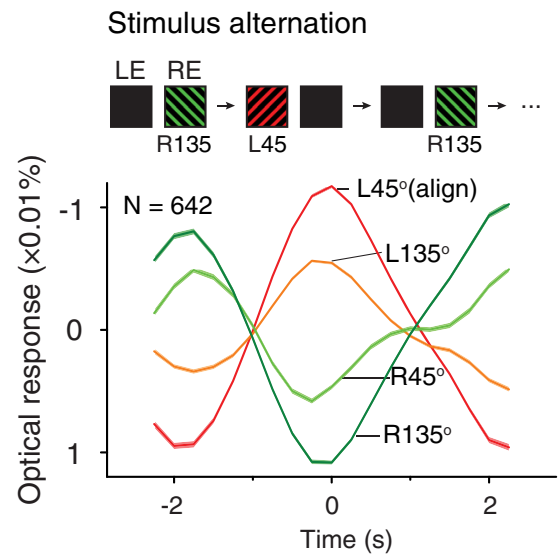

C
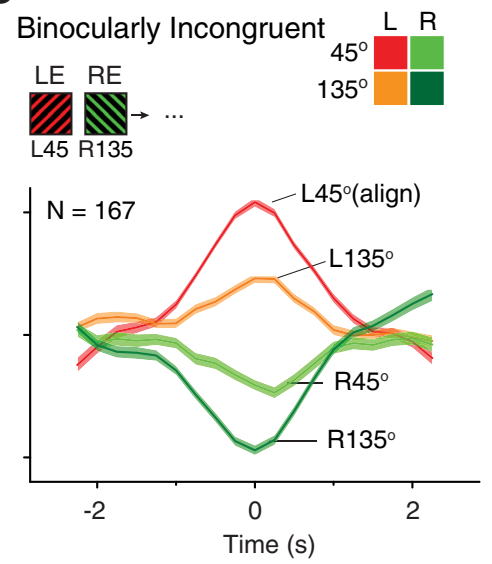

Figure 5. Responses in different orientation domains. $A$, An orientation map obtained with 45 and $135^{\circ}$ gratings. The magnified panel shows outlines of the orientation domains preferring $45^{\circ}$ (dotted yellow outlines) and $135^{\circ}$ (yellow outlines) orientations. Red and green outlines denote left- and right-eye columns (same as in Fig. 1B). $\boldsymbol{B}$, Mean responses ( \pm SEM) of four types of domains in stimulus-alternation conditions, aligned with peaks in the $L 45$ domain time courses. $\boldsymbol{C}$, Similar to $\boldsymbol{B}$, responses to $B$ stimuli aligned with $L 45$ peaks. Lu, Lunate sulcus.

19-frame-long (4.75 s) $d R / R$ frame series centered at these time points. These frame series were then averaged across all trials. From this averaged frame series, pixel-averaged response curves from different functional compartments were obtained. For nine types of functional domains (see above, Functional domain identification), we obtained nine averaged responses (Fig. 6A, simplified version in Fig. 5B). Similarly, we also calculated L45-trough-aligned responses (Fig. 6B). For L135R45 stimulusalternation data, we used peaks/troughs of L135 domains for alignment (Fig. $6 C, D$ ). The same analysis was applied to all BI data (Fig. $6 E-H$, simplified version in Fig. $5 C$ ). For BI data, the detected peaks/troughs had a wide distribution of dominance durations (Fig. 2B). To achieve a good signal-noise ratio in their average responses, only peak/trough durations between 1.5 and $6 \mathrm{~s}$ were included in this analysis.

Pattern classification approach. Similar to its application in fMRI studies (Haynes and Rees, 2005; Kamitani and Tong, 2005), a multivariate pattern classification based on a support vector machine (SVM) was used as an alternative approach in evaluating the $\mathrm{V} 1$ responses during $\mathrm{BI}$ stimulation. This approach classifies an input frame based on learned pattern differences between two groups of training frames obtained in monocular stimulation imaging. Thus, it does not rely on the explicit knowledge of the V1 OD patterns. This classification was performed using the LIBSVM implementation (Chang and Lin, 2011), which has been shown to be effective in optical imaging data analysis (Xiao et al., 2008). To train a pattern classifier for a specific BI condition, V1 responses to two monocular half images were first imaged. The half images were the same as those in the BI condition except that each time only one half image was presented to one eye. For two BI conditions (L45R135 and
L135R45), a total of four training conditions were imaged (L45, R135, L135, R45). First we used a linear SVM kernel and threefold crossvalidation to search for the best parameter $C$ (Chang and Lin, 2011; Xiao et al., 2008). C was the only parameter for linear kernel SVM that controlled the error tolerance. The typical classification accuracy for cross-validation was above $99 \%$, with a $C$ of $2^{-10}$. The SVM was then trained with all the training data. In test session, the SVM calculated a single number for each input "unknown" frame, an OD score, which depicted how likely it was (the probability) the input frame belonged to one of the two training classes.

Analysis of color balance data. To examine the dependence of BR on stimulus strength, the color balance data (described above, see Color balance test) were further analyzed quantitatively (Fig. 2C,D). We first obtained L-R time courses for all $4 \mathrm{~s}$ trials (Fig. 2C). A trialaveraged L-R value (or R-L value for L135R45 data) from frames $4-18$ were used to represent the eye-dominance bias for one particular red/ green pair (each dot in Fig. 2D). The population color balance data was then fitted with a linear function.

Single-trial videos. Single-trial videos (Movies 1,2) were created based on $d R / R$ frames in single BI trials. A video frame was an average of 3 consecutive imaging frames (total $0.75 \mathrm{~s}$ ) and with a local average of 49 neighboring frames (12 s) subtracted. A subsequent filtering (low-pass and high-pass filters; disk kernel size, 2 and 50 pixels, respectively) was applied and the result images were clipped at 1.5 SD. In Movie 2, for better illustration and also comparison with the standard OD patterns, red and green outlines, corresponding to the left-eye and right-eye OD columns, were superimposed onto the video frames near each monocular dominance phases. For detailed examination, these frames were played at a slower $(0.8 \times)$ speed, whereas other frames were played at a $4 \times$ speed.

\section{Results}

We used intrinsic signal optical imaging methods (Ts'o et al., 1990; Li et al., 2013) and imaged responses to binocularly incongruent stimuli through an optical chamber over V1. Our stimuli consisted of two dichoptic patches (half images) of $45^{\circ}$ (red) or $135^{\circ}$ (green) drifting gratings presented on each side of a CRT screen, converged by a pair of prisms to form a fused binocular view (Fig. 1A). To quantify responses in V1 eye-specific columns, V1 OD maps were obtained by comparing cortical responses to grating stimuli presented monocularly (Fig. 1B). Based on the pixel gray values, left-eye and right-eye columns were determined (Fig. $1 B$, inset).

To study responses to BI stimuli in anesthetized animals, we first examined whether with a single-trial analysis eyedominance state could be determined in a "stimulusalternation" paradigm, during which two half images were in turn presented to each eye alone at a fixed rate $(0.25 \mathrm{~Hz})$. Each trial lasted $60 \mathrm{~s}$, and cortical responses were imaged continuously at a frame rate of $4 \mathrm{~Hz}$. Figure $1 C$ shows from an example trial the pixel-averaged time courses calculated by averaging the reflectance change values for all left-eye (red) or right-eye (green) pixels within the stimulus activated V1 region. The 

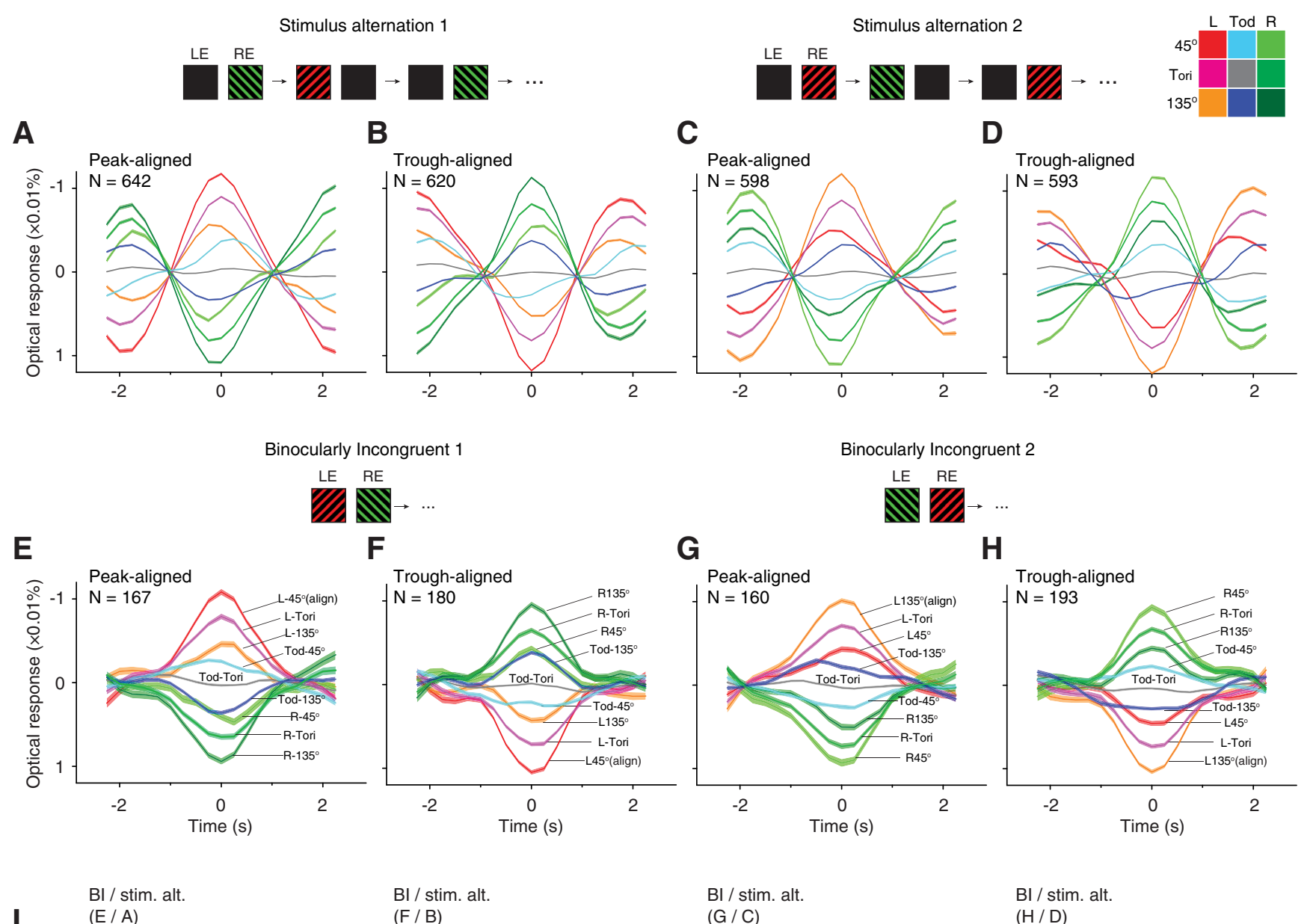

$\mathrm{BI} /$ stim. alt.
(F/B)

BI / stim. alt.

$(\mathrm{G} / \mathrm{C})$

BI / stim. alt.

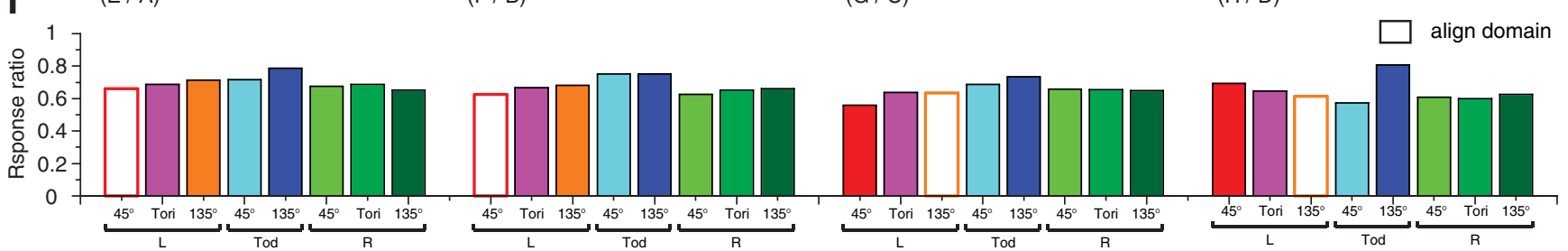

Figure 6. Comparison of fluctuation amplitudes in stimulus-alternation and BI trials. $A-D$, Similar to Figure $5 B$, aligned and averaged stimulus-alternation responses in nine functional compartments (color code in top right corner). $\boldsymbol{A}, \boldsymbol{B}$, Response to $\mathrm{L} 45 \mathrm{R} 135$ stimulus-alternation aligned with $\mathrm{L} 45$ peaks $(\boldsymbol{A})$ or troughs $(\boldsymbol{B})$. $\boldsymbol{C}, \boldsymbol{D}, \operatorname{Similar}$ to $\boldsymbol{A}$ and $\boldsymbol{B}$ but for $\mathrm{L} 135 \mathrm{R} 45$ stimuli. $\boldsymbol{E}-\boldsymbol{H}$, Similar to Figure 5 , aligned BI responses in nine functional compartments. $\boldsymbol{E}, \boldsymbol{F}$, Averaged domain responses to $L 45 R 135 \mathrm{BI}$ stimuli, aligned with $L 45$ peaks $(\boldsymbol{E})$ or troughs $(\boldsymbol{F})$. $\boldsymbol{G}, \boldsymbol{H}$, Similar to $\boldsymbol{E}$ and $\boldsymbol{F}$ but for L135R45 BI stimuli, aligned with L135 peaks $(\boldsymbol{G})$ or troughs $(\boldsymbol{H})$. Note that except for the widths of the cycles, the response profiles for BI stimuli are generally similar to those in corresponding stimulus-alternation results. All curves were plotted as mean \pm SEM. $I$, Response amplitude ratios for BI and corresponding stimulus-alternation curves. Labels above each panel indicate from which two panels the ratios were calculated. The response amplitudes for $\mathrm{T}_{0 D} \mathrm{~T}_{\text {ori }}$ domains were too small to obtain meaningful ratios, and thus were not plotted. On average, the peak amplitudes for $\mathrm{BI}$ stimulus were $67.4 \%$ of those in the stimulus-alternation conditions.

cyan curve represents the difference between these two monocular curves (L-R signal). The two monocular curves exhibited a periodical counterphase modulation, which matches the stimulus alternation (dotted lines). With continuous imaging and this single-trial analysis, the optical response signals were found to quickly follow the stimulus alternations.

From five hemispheres of four monkeys, we imaged 128 stimulus-alternation trials. L-R time course power spectrums (Fig. $1 D$ ) have a clear peak at the stimulus frequency $(0.25 \mathrm{~Hz})$. In addition, the phase differences between the left- and right-eye phase spectrums are dominated by $180^{\circ}$ phase differences (Fig. $1 E$, yellow). These positive results confirmed that the V1 eyedominance information can be measured from single-trial optical imaging frames without trial averaging, enabling the examination of the eye-dominance state during BI stimulation. If rivalry does occur in anesthetized V1, we should be able to observe fluctuations in eye-specific columns.

So what happens in anesthetized V1 during BI stimulation? The same analysis was applied to the BI trials collected when the two eyes were viewing two different half images simultaneously. An example BI trial is shown in Figure $1 F$, where the left eye was viewing a red $45^{\circ}$ grating while the right eye was viewing a green $135^{\circ}$ grating. The measured left- and right-eye signals fluctuated over the entire imaging period, evidently in a counterphase fashion. Unlike the fast, regular fluctuations in the stimulus-alternation condition, the fluctuations in the BI condition appeared slower and had irregular durations. We collected $148 \mathrm{BI}$ trials from five hemispheres. Population results are shown in Figure 1, $G$ and $H$. The power spectrums of the $\mathrm{L}-\mathrm{R}$ time courses demonstrated a distributed energy in 


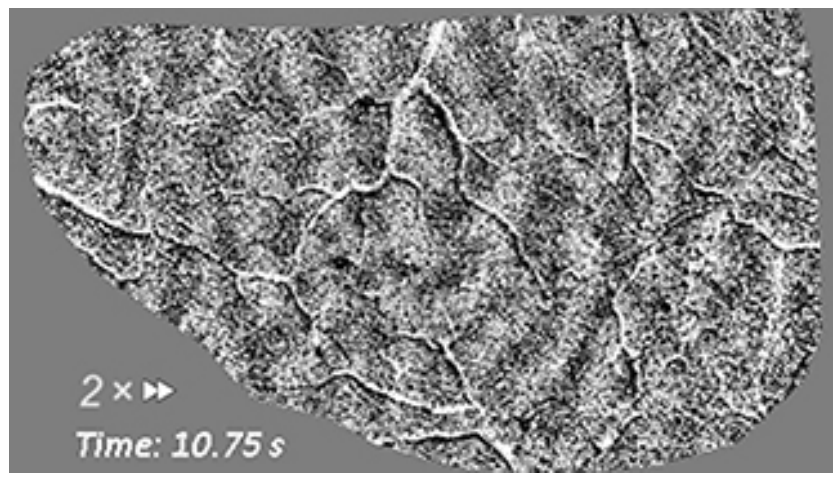

Movie 1. V1 eye-specific activation fluctuates during Bl stimulation. This movie was clipped from an example single-trial imaging of $\mathrm{V} 1$ responses during $\mathrm{BI}$ stimulation. The original imaging frames were first transformed to $d R / R$ frames and spatially filtered (see Materials and Methods). Each video frame shown here is an average of 3 neighboring imaging frames (total of $0.75 \mathrm{~s}$ ), subtracting the average of 49 neighboring frames ( $12 \mathrm{~s})$. The video was played at $2 \times$ speed.

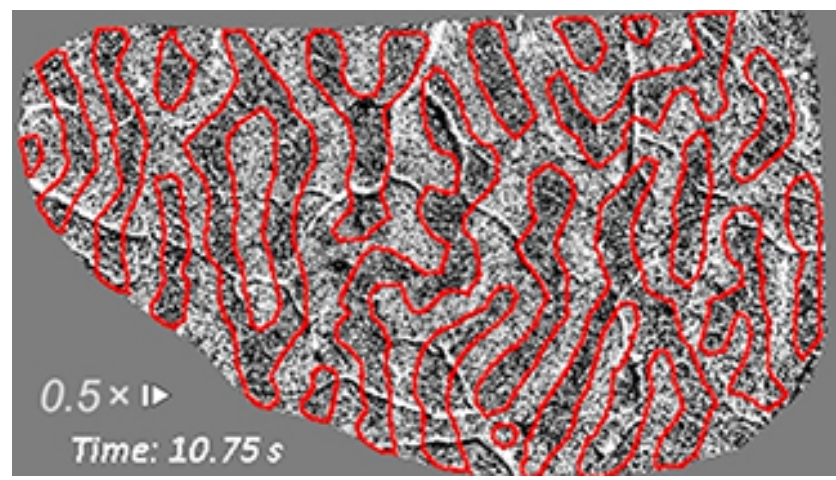

Movie 2. Same data as Movie 1 with annotations. The movie shows the same data as in Movie 1 played at $4 \times$ speed and slowed down to $0.8 \times$ speed when an 0D pattern emerges. Left-eye OD outlines (red) and right-eye OD outlines (green) are superimposed to facilitate identification of these domains.

$0.03 \sim 0.2 \mathrm{~Hz}$ (Fig. $1 G$ ), where counterphase modulations were also observed (Fig. $1 H$ ).

For comparison, we also imaged V1 activation when the two eyes were viewing stimuli that normally do not cause BR, for example, identical plaid patterns or random dot patterns. In the plaid condition, each plaid was a simple combination of the two half images used in the BI condition. In strong contrast to the BI trials, the plaid time courses were largely flat (Fig. 1I). Frequency analysis of the plaid $(n=25)$ and $\mathrm{RD}(n=49)$ trials showed much weaker low-frequency power compared with the BI trials (Fig. $1 \mathrm{~J}$ ), as well as a lack of counterphase eye modulations (Fig. $1 K$ ). The trial-averaged power spectrums (Fig. 1L) show a much lower power for plaid/RD at low frequencies. Figure $1 M$ shows the trial-averaged phasespectrums, both stimulus-alternation and BI had a mean phase differences close to $135^{\circ}$, whereas the values for plaid/RD conditions were around $90^{\circ}$, the mean result from a random distribution between $0-180^{\circ}$.

The above pixel-averaging analysis relies on prior knowledge of the V1 OD maps. We also analyzed the data using an alternative approach of multivariate pattern classification. The results obtained from these two methods were very similar. Mean correlation coefficient for BI time courses obtained from the two different methods was $0.76 \pm 0.02$, and that for stimulus alternation was $0.82 \pm 0.01$.
Counterphase alternations can also be observed directly in single-trial images. An example trial is shown in Movies 1 and 2. Figure $2 A$ shows 10 representative images extracted from the video at locations where the $\mathrm{L}-\mathrm{R}$ time course reached peaks or troughs. Each image is an average of neighboring three imaging frames and subtracting of a $12 \mathrm{~s}$ baseline. These two sets of images show complementary patterns that match the left-eye (red transparent shades) and right-eye (green transparent shades) OD columns imaged in a separate monocular stimulation run (superimposed on the first images).

Are the observed V1 OD alternations really BR? We further examined the alternations for key features of BR. One is the distribution of perceptual eye dominance durations, which can be fitted with a gamma distribution (Levelt, 1967; Leopold and Logothetis, 1996). From all BI L-R time courses, we identified 664 peaks and troughs. The distribution of these V1 eye dominance durations were well fitted by a gamma function skewed to the left (Fig. 2B), consistent with those obtained in psychophysics (Levelt, 1967) and animal behavior (Leopold and Logothetis, 1996) observations. The mean dominance duration was $4.3 \mathrm{~s}$, which is longer than the mean duration reported for awake monkeys (2.3 s) (Leopold and Logothetis, 1996). Differences in these two mean dominance durations could be due to experimental factors, including awake/anesthetized differences (e.g., lack of top-down attention, stable eye positions, etc.), stimulus differences, and measuring methods (imaging vs behavior).

Another feature of BR is that the distribution of eye dominance durations is readily biased by stimulus strength (Levelt, 1965). As a part of each experiment, we tested a series of redgreen luminance combinations to obtain a balanced pair for the two eyes. Based on data from these "color balance tests" we examined how eye dominance was modulated by stimulus strength. The stimulus was similar to that used in the BI condition, except that we increased or decreased the luminance level of one half image and kept the other half image unchanged. The imaging time was $4 \mathrm{~s}$ to allow increased numbers of stimulus conditions. Figure $2 C$ shows the $\mathrm{L}-\mathrm{R}$ time courses obtained in three stimulus conditions in which the luminance of the red grating was set at three different levels. When the left eye was presented with a low luminance red grating, the V1 activation showed a bias toward right-eye dominance (left panel). This bias was reversed to lefteye dominance when the luminance of the left-eye grating was increased (right panel). Between these two ends there is a balanced red/green luminance ratio, at which the eye dominance was equally distributed to the two eyes (middle panel). Importantly, during this balanced condition, the L-R signal of each individual trial did not remain at 0 ; instead, it showed either left-eye-biased or right-eye-biased responses. This is consistent with $\mathrm{BR}$ perception, where subjects mostly see rivaling monocular images. For each panel in Figure $2 C$, we calculated the mean $\mathrm{L}-\mathrm{R}$ values over the response period (frames $4-18$ ). Figure $2 D$ shows such values obtained in all tests and their linear fitting. Within individual and across different monkeys, V1 eye dominance showed a predictable shift when the stimulus strength was modulated, which parallels the observations in rivalry perception (Levelt, 1965; Leopold and Logothetis, 1996).

In addition to stimulus strength, spatial context of the BR stimuli also plays an important role in modulating the eye dominance. For example, psychophysical studies have shown that dominance during $\mathrm{BR}$ is predisposed to the rivaling image with a $\mathrm{MBC}$ (Ooi and He, 2006). If neural events important for the occurrence of BR arise within $\mathrm{V} 1$, then such a contextual modu- 
lation should also be observed in the same condition. Here, we tested this hypothesis in anesthetized monkey V1.

Similar to the MBC stimuli used in previous psychophysics studies (Ooi and He, 2006), both half images had the same $7 \times 7^{\circ}$ black-white grating background (Fig. $3 A$ ). One half image contained a $2^{\circ}$-diameter grating disk in the center whose orientation was orthogonal to its grating background. Thus, the two half images possessed a rival component (orthogonal orientation) at the central disk and a nonrival component outside the disk. We examined the eye-dominance responses in the V1 regions corresponding to the central disk by calculating its $\mathrm{L}-\mathrm{R}$ signal. Figure $3 A$ shows the $\mathrm{L}-\mathrm{R}$ time courses for four stimulus conditions imaged in one experiment. Both individual trials (gray curves) and their average (red curves) show a response bias toward the eye viewing a boundary contour (two-way ANOVA showed significant effect for eye of origin, $\left.F_{(1,96)}=158.04, p<0.001\right)$. Biased eye dominance was best visualized in maps where frames from the left-eye-MBC and right-eye-MBC conditions were compared (Fig. $3 B$ ). The map contains a clear OD pattern at the retinotopic location corresponding to the monocular disk in the stimuli (retinotopic location confirmed in Fig. 3C). Such a MBC-induced OD pattern was observed in all cases imaged (Fig. 4). Thus, the V1 alternation is modulated by the spatial context during BI in a way parallels the human psychophysical observations (Ooi and $\mathrm{He}$, 2006). The similarities between monkey V1 responses and human psychophysics further indicate that V1 plays an important role in BR perception.

Thus far, by examining V1 OD signals, we have demonstrated the existence of several important features of the rivalry-like alternations in anesthetized monkey V1. However, it is still unknown how orientation information contributed to this process. To evaluate orientation signals, we identified 45 and $135^{\circ}$ orientation domains based on 45 versus $135^{\circ}$ orientation maps (Fig. $5 A$ ) and constructed four types of OD orientation domains (L45, L135, R45, and R135).

We first examined responses in stimulus-alternation conditions. Based on pixel-averaged time courses, we aligned and averaged all L45 peaks into one curve, and the responses in the other three types of domains were coaligned (i.e., L45-peak triggered average; Fig. $5 B$ ). As expected, all four curves show a 4 s/cycle modulation. When responses in L45 domains reached a peak during the left-eye $45^{\circ}$ stimulation, R135 domains exhibited lowest responses likely due to the removal of the right-eye $135^{\circ}$ stimulation and/or the appearance of the left-eye $45^{\circ}$ stimulation. For L135 and R45 domains, which receive incongruent influences from the eye-of-origin and orientation inputs, the responses appeared to be primarily determined by the eye-of-origin inputs: L135 domains showed a response increase when the stimulus was left-eye $45^{\circ}$ gratings, whereas $\mathrm{R} 45$ domains showed a response decrease.

For BI data analyzed with the same methods (Fig. 5C), the relative response amplitudes were very similar to those in the stimulus-alternation condition. When L45 domains reached a peak, R135 domains exhibited the lowest responses. Since in this BI condition the R135 half image was constantly present, the decrease of responses in R135 domains can no longer be attributed to the stimulus changes. Meanwhile, L135 and R45 responses still appeared to be dominated by the eye-of-origin influences, not by the orientation influences. Although orientation alternations were also present (e.g., differences between L45 and L135 domains), they were much weaker than the OD alternations (e.g., differences between L45 and R45 domains). This is also evident in the patterns observed in Movies 1 and 2: the emerged patterns were evidently OD patterns, not orientation patterns. A more complete analysis is shown in Figure 6 in which the trends described above still hold. Together, the overall similarity between BI and stimulus-alternation response patterns indicate that the "suppressed" half image contributed very little to the V1 responses. However, we should also note that many features in stimulus-alternation conditions (e.g., abrupt appear/disappear, fixed alternation frequency) were very different from those in rivalry perceptions. A better comparison can be achieved by using "replay" type of stimulus that mimics the stochastic events occurring in the BI condition.

\section{Discussion}

With high-resolution optical imaging and single-trial analysis methods, we have provided direct evidence of rivalry-like neural activity in anesthetized subjects. Although it remains to be demonstrated that such neural activity indeed underlies conscious BR perception, the alternations observed in this study exhibit many of the characteristic features of BR observed in psychophysical and neural physiological studies. Our anesthetized preparation also provided several unique advantages for this type of studies. First, one does not need to worry about conscious-related influences, or extra signals generated by introspection or active reporting (e.g., Frässle et al., 2014). Second, compared with awake subjects, anesthetized preparations provide more stable eye positions, no eye blinks, longer imaging time and a lower noise level, which were all important for the successful single-trial analysis in this study. There are also some limitations in using intrinsic signal imaging in studying BR, for example, slow hemodynamic signal limited the detection of fast alternations and cortical waves.

\section{Binocular rivalry and attention}

In the past nearly two centuries, many studies investigated the necessity and effects of attention on $\mathrm{BR}$, and the results have been controversial (Dieter and Tadin, 2011; Paffen and Alais, 2011). Psychophysical studies show different degrees of attentional effects on BR perception that range from minor (Ooi and He, 1999; Mitchell et al., 2004) to complete suppression (Brascamp and Blake, 2012). Measured with fMRI or EEG techniques, passive and attentive viewing shows similar BR activity in occipital cortex (Frässle et al., 2014), while attention directed elsewhere decreases (Lee et al., 2007; Zhang et al., 2011) or increases (Roeber et al., 2011) the ongoing rivalry activity in early visual cortex. Importantly, a basic question is still under debate: whether attention is required for BR to occur? The difficulty of fully addressing this seemingly simple question is probably due to the complex effects in various ways of modulating subjects' attention in the previous studies. For example, it is difficult to assess how much attention still exists in the passive-viewing conditions, or whether BR is actively suppressed in the attend-away conditions. Here, the anesthetized preparation provides a unique experimental paradigm where voluntary controls were completely removed. Our results from anesthetized monkeys suggest that low-level automatic mechanisms alone are capable of initiating BR. In addition to the existence of rivalry alternation, we further show that several basic features of BR perception are not dependent on conscious processes and can be observed in early stages of visual processing in anesthetized conditions. Thus, although voluntary attention can influence BR to various extends, it is not a necessary component for BR.

Top-down influences also include mechanisms that do not rely on consciousness. These mechanisms include, for example, figure-ground segregation, perceptual grouping, and rivalry sig- 
nals that develop or strengthen at higher visual areas, many of which may persist in anesthetized conditions and could play a role in the rivalry-like activity we observed in V1. However, considering that these signals are mostly binocular, and we observe stronger monocular alternations in V1, these top-down mechanisms are unlikely the main causes of the fluctuations we observed. In our experimental conditions, it appears that more assumptions would be needed to suggest that V1 BR is due to top-down influences than being locally generated.

Previous fMRI studies showed that human frontal-parietal cortex is actively involved in the perception of bistable switches as compared with physical stimulus switches (Lumer et al., 1998; Sterzer and Kleinschmidt 2007; Weilnhammer et al., 2013). It was thus suggested that these cortical regions may play a central role in initiating the alternations during bistable perception (Leopold and Logothetis, 1999). However, several previous studies showed that by removal of introspection and awareness of the perceptual switches, the frontal-parietal activation was no longer significant (Knapen et al. 2011; Frässle et al., 2014; Brascamp et al., 2015). These latter results suggest that the activation in frontal-parietal cortex is actually the consequences of the perceptual switches instead of their causes. So where the switches initiate if it is not the frontal-parietal cortex? It is conceivable that the switches may arise from neural processes earlier in the visual pathways. Here our results show that automatic alternations of V1 OD patterns occur in the absence of conscious visual awareness. If the same neural processes occur in awake subjects, then this would be consistent with the initiation role of V1 in BR.

\section{Eye rivalry versus pattern rivalry}

What are the underlying neural mechanisms contributed to the observed V1 alternations? Much physiological evidence suggests that interocular inhibition could contribute to such neuronal phenomenon (Varela and Singer 1987; Sengpiel and Blakemore 1994; Sengpiel et al. 2001; Bahmani et al. 2014). However, interocular inhibition, or eye rivalry, cannot account for many BR observations, for example, stimulus rivalry or pattern rivalry (Kovács et al. 1996; Logothetis et al., 1996). In these conditions, rivalry competitions appear to occur after binocular convergence and between neurons coding different patterns. In addition, in their single-cell recordings from awake monkeys, Leopold and Logothetis (1996) found only a small percentage of monocular neurons that were modulated by monkey's rivalry perception (see also the discussion in the next section). Thus, neither of these two theories alone can fully account for the experimental findings. BR is likely a series of processes that different mechanisms may involve at different levels (Blake and Logothetis 2002). Our observations in $\mathrm{V} 1$ are more consistent with the eye-based rivalry: during BI stimulation, the overall patterns fluctuated in V1 were mainly OD patterns, instead of orientation patterns (Fig. 2A, Movies 1 and 2). Also, when specific eye-orientation domains received incongruent eye-pattern information, their activity was mainly driven by the eye-specific information, not by the orientation information (Fig. 5C, R45, L135 domains).

Interestingly, we also observed similar alternations in V2 orientation domains, which were in-phase with the $\mathrm{V} 1$ orientation alternations (data not shown). Since the sizes of exposed V2 were relatively small (Fig. 5A) and the signal was noisier (close to the large blood vessels on the lunate sulcus), this result was only suggestive. It would be very interesting to study the temporal relationships between these V1 and V2 in-phase modulations, which was challenging here based on the slow intrinsic optical signals.

\section{Monkey electrophysiology versus human fMRI}

Previous fMRI imaging in humanV1 (Polonsky et al., 2000; Tong and Engel, 2001) has shown strong BR modulation. However, single-cell recordings from awake monkeys found that only a small percentage of neurons in V1/V2 responded in a way coincident with monkeys' BR perception (Leopold and Logothetis, 1996; Keliris et al. 2010). Monkey LFP and human EEG results are more like the fMRI ones (Maier et al. 2008; Zhang et al. 2011). Such monkey electrophysiology versus human fMRI discrepancies were also found in other types of cognitive tasks, such as attention (Boynton, 2011; Watanabe et al., 2011). It is unclear whether species differences or signal differences underlie these discrepancies (Boynton, 2011). A previous study combined fMRI and electrophysiology in awake monkeys and provided important evidence to narrow down the causes to differences in the nature of the signals (Maier et al., 2008). In our study, intrinsic optical signal is mainly hemodynamic based, which is closer to fMRI signals. Similar to fMRI findings in humans (Polonsky et al., 2000; Tong and Engel, 2001), we found strong rivalry-like hemodynamic activity in V1 responses. This is consistent with the suggestion that the monkey electrophysiology versus human fMRI controversy is due to the nature of the signals, instead of species differences. There are many possibilities that may cause such divergence in signals. For example, single-cell recordings may have some systematic bias to certain groups of neurons and undersample neurons that exhibit stronger modulations during perceptual changes. Or, higher-level feedback is more represented in subthreshold neural activity, which causes more modulations in the hemodynamic signal, but not in the spike signal. Our parallel findings from anesthetized animals make the second hypothesis less likely and the first one (biased electrophysiology recording) more likely.

In summary, we showed clear evidence that rivalry-like alternations persist in subjects under anesthesia. This indicates that low-level automatic mechanisms play an active role in solving the problem of visual ambiguity, in a way that cooperates with, but does not rely on, conscious influences.

\section{References}

Alais D, Blake R (2005) Binocular rivalry. Cambridge, MA: MIT.

Bahmani H, Murayama Y, Logothetis NK, Keliris GA (2014) Binocular flash suppression in the primary visual cortex of anesthetized and awake macaques. PLoS One 9:e107628. CrossRef Medline

Blake R, Logothetis N (2002) Visual competition. Nat Rev Neurosci 3: 13-21. CrossRef Medline

Boynton GM (2011) Spikes, BOLD, attention, and awareness: a comparison of electrophysiological and fMRI signals in V1. J Vis 11(5):12 1-16. CrossRef Medline

Brascamp JW, Blake R (2012) Inattention abolishes binocular rivalry: perceptual evidence. Psychol Sci 23:1159-1167. CrossRef Medline

Brascamp J, Blake R, Knapen T (2015) Negligible fronto-parietal BOLD activity accompanying unreportable switches in bistable perception. Nat Neurosci 18:1672-1678. CrossRef Medline

Chang CC, Lin CJ (2011) LIBSVM: a library for support vector machines. ACM Trans Intell Syst Technol 2:1-27. CrossRef

Dieter KC, Tadin D (2011) Understanding attentional modulation of binocular rivalry: a framework based on biased competition. Front Hum Neurosci 5:155. Medline

Frässle S, Sommer J, Jansen A, Naber M, Einhäuser W (2014) Binocular rivalry: frontal activity relates to introspection and action but not to perception. J Neurosci 34:1738-1747. CrossRef Medline

Friman O, Borga M, Lundberg P, Knutsson H (2004) Detection and detrending in fMRI data analysis. Neuroimage 22:645-655. CrossRef Medline

Haynes JD, Rees G (2005) Predicting the stream of consciousness from activity in human visual cortex. Curr Biol 15:1301-1307. CrossRef Medline 
Kamitani Y, Tong F (2005) Decoding the visual and subjective contents of the human brain. Nat Neurosci 8:679-685. CrossRef Medline

Keliris GA, Logothetis NK, Tolias AS (2010) The role of the primary visual cortex in perceptual suppression of salient visual stimuli. J Neurosci 30: 12353-12365. CrossRef Medline

Knapen T, Brascamp J, Pearson J, van Ee R, Blake R (2011) The role of frontal and parietal brain areas in bistable perception. J Neurosci 31: 10293-10301. CrossRef Medline

Kovács I, Papathomas TV, Yang M, Fehér A (1996) When the brain changes its mind: Interocular grouping during binocular rivalry. Proc Natl Acad Sci U S A 93:15508-15511. CrossRef Medline

Lee SH, Blake R, Heeger DJ (2007) Hierarchy of cortical responses underlying binocular rivalry. Nat Neurosci 10:1048-1054. CrossRef Medline

Leopold DA, Logothetis NK (1996) Activity changes in early visual cortex reflect monkeys' percepts during binocular rivalry. Nature 379:549-553. CrossRef Medline

Leopold DA, Logothetis NK (1999) Multistable phenomena: changing views in perception. Trends Cogn Sci 3:254-264. Medline

Levelt WJ (1965) On binocular rivalry. PhD dissertation. Institute of Perception, Soesterberg, The Netherlands. Available at: http://www.mpi.nl/world/ materials/publications/levelt/Levelt_Binocular_Rivalry_1965.pdf.

Levelt WJM (1967) Note on the distribution of dominance times in binocular rivalry. Br J Psychol 58:143-145. CrossRef Medline

Li P, Zhu S, Chen M, Han C, Xu H, Hu J, Fang Y, Lu HD (2013) A motion direction preference map in monkey V4. Neuron 78:376-388. CrossRef Medline

Logothetis NK, Leopold DA, Sheinberg DL (1996) What is rivalling during binocular rivalry? Nature 380:621-624. CrossRef Medline

Lu HD, Chen G, Ts'o DY, Roe AW (2009) A rapid topographic mapping and eye alignment method using optical imaging in Macaque visual cortex. Neuroimage 44:636-646. CrossRef Medline

Lumer ED, Friston KJ, Rees G (1998) Neural correlates of perceptual rivalry in the human brain. Science 280:1930-1934. CrossRef Medline

Maier A, Wilke M, Aura C, Zhu C, Ye FQ, Leopold DA (2008) Divergence of fMRI and neural signals in V1 during perceptual suppression in the awake monkey. Nat Neurosci 11:1193-1200. CrossRef Medline

Mitchell JF, Stoner GR, Reynolds JH (2004) Object-based attention determines dominance in binocular rivalry. Nature 429:410-413. CrossRef Medline

Ooi TL, He ZJ (1999) Binocular rivalry and visual awareness: the role of attention. Perception 28:551-574. CrossRef Medline

Ooi TL, He ZJ (2006) Binocular rivalry and surface-boundary processing. Perception 35:581-603. CrossRef Medline
Paffen CL, Alais D (2011) Attentional modulation of binocular rivalry. Front Hum Neurosci 5:105. Medline

Pichlmayr I, Lehmkuhl P, Lips U (1987) EEG atlas for anesthesiologists. Berlin: Springer.

Polonsky A, Blake R, Braun J, Heeger DJ (2000) Neuronal activity in human primary visual cortex correlates with perception during binocular rivalry. Nat Neurosci 3:1153-1159. CrossRef Medline

Roeber U, Veser S, Schröger E, O'Shea RP (2011) On the role of attention in binocular rivalry: electrophysiological evidence. PLoS One 6:e22612. CrossRef Medline

Sengpiel F, Blakemore C (1994) Interocular control of neuronal responsiveness in cat visual cortex. Nature 368:847-850. CrossRef Medline

Sengpiel F, Freeman TCB, Bonhoeffer T, Blakemore C (2001) On the relationship between interocular suppression in the primary visual cortex and binocular rivalry. Brain Mind 2:39-54. CrossRef

Sterzer P, Kleinschmidt A (2007) A neural basis for inference in perceptual ambiguity. Proc Natl Acad Sci U S A 104:323-328. CrossRef Medline

Tanabe J, Miller D, Tregellas J, Freedman R, Meyer FG (2002) Comparison of detrending methods for optimal fMRI preprocessing. Neuroimage 15: 902-907. CrossRef Medline

Tong F, Engel SA (2001) Interocular rivalry revealed in the human cortical blind-spot representation. Nature 411:195-199. CrossRef Medline

Ts'o DY, Frostig RD, Lieke EE, Grinvald A (1990) Functional organization of primate visual cortex revealed by high resolution optical imaging. Science 249:417-420. CrossRef Medline

Varela FJ, Singer W (1987) Neuronal dynamics in the visual corticothalamic pathway revealed through binocular rivalry. Exp Brain Res 66:10-20. Medline

Watanabe M, Cheng K, Murayama Y, Ueno K, Asamizuya T, Tanaka K, Logothetis N (2011) Attention but not awareness modulates the BOLD signal in the human V1 during binocular suppression. Science 334: 829-831. CrossRef Medline

Weilnhammer VA, Ludwig K, Hesselmann G, Sterzer P (2013) Frontoparietal cortex mediates perceptual transitions in bistable perception. J Neurosci 33:16009-16015. CrossRef Medline

Xiao Y, Rao R, Cecchi G, Kaplan E (2008) Improved mapping of information distribution across the cortical surface with the support vector machine. Neural Networks 21:341-348. CrossRef Medline

Yang Z, Heeger DJ, Blake R, Seidemann E (2015) Long-range traveling waves of activity triggered by local dichoptic stimulation in V1 of behaving monkeys. J Neurophysiol 113:277-294. CrossRef Medline

Zhang P, Jamison K, Engel S, He B, He S (2011) Binocular rivalry requires visual attention. Neuron 71:362-369. CrossRef Medline 